\title{
FINITE ELEMENT ANALYSIS OF THE RANDOM RESPONSE SUPPRESSION OF COMPOSITE PANELS AT ELEVATED TEMPERATURES USING SHAPE MEMORY ALLOY FIBERS
}

\author{
Travis L. Turner ${ }^{\dagger}$ \\ NASA Langley Research Center \\ Hampton, VA 23681-0001 \\ Z. W. Zhong and Chuh Mei* \\ Old Dominion University \\ Norfolk, VA 23529-0247
}

\begin{abstract}
A feasibility study on the use of shape memory alloys (SMA) for suppression of the random response of composite panels due to acoustic loads at elevated temperatures is presented. The constitutive relations for a composite lamina with embedded SMA fibers are developed. The finite element governing equations and the solution procedures for a composite plate subjected to combined acoustic and thermal loads are presented. Solutions include: 1) critical buckling temperature, 2) flat panel random response, 3) thermal postbuckling deflection, and 4) random response of a thermally buckled panel. The preliminary results demonstrate that the SMA fibers can completely eliminate the thermal postbuckling deflection and significantly reduce the random response at elevated temperatures.
\end{abstract}

\section{Introduction}

Future high-performance aircraft, such as the high speed civil transport (HSCT), will subject the external skin panels to significant acoustic and thermal loads. The new vectored thrust propulsion systems on the

$\dagger \quad$ Aerospace Engineer, Structural Acoustics Branch, Acoustics Division, Member AIAA.

‡ Graduate Research Assistant, Center for Structural Acoustics and Fatigue Research, Aerospace Engineering Department, Student Member AIAA.

* Professor, Center for Structural Acoustics and Fatigue Research, Aerospace Engineering Department, Associate Fellow AIAA.

Copyright (c) 1994 by the American Institute of Aeronautics and Astronautics, Inc. No copyright is asserted in the United States under Title 17, U.S. code. The U.S. Government has a royalty-free license to exercise all rights under the copyright claimed herein for Government purposes. All other rights are reserved by the copyright owner.
YF-22 Advanced Tactical Fighter ATF provide short take-off and landing capability and increased maneuverability. However, the vectored thrust also subjects portions of the aircraft structure to intensified acoustic and thermal loads. Acoustic fatigue thus emerges as one of the critical considerations in the design and development of high speed flight vehicles ${ }^{1}$.

A significant amount of research in the last few years has greatly advanced the field of control of flexible structures through the use of smart materials ${ }^{2}$. The field of adaptive or intelligent structures involves a broad range of technologies that enable the realization of structural systems that are able to sense and to control their own behavior, such that the range of operational performance for these materials may be extended over conventional materials and structures. Smart materials have the ability to change shape, stiffness, natural frequency, damping, and other mechanical characteristics in response to changes in temperature, electric field, or magnetic field. The most common smart materials are shape memory alloys, piezoelectric materials, magnetostrictive materials, electrorheological fluids and magnetorheological fluids.

\section{Shape Memory Alloy Hybrid Composites}

Shape memory alloys exhibit a characteristic phase transformation from martensite to austenite, initiating at the austenite start temperature $T_{s}$ and asymptotically ending at the austentite finish temperature $T_{f}$. A shape memory alloy in the low temperature martensitic condition $\left(T<T_{s}\right)$, when plastically deformed and the external stresses removed, will regain its original (memory) shape when heated. For example, strains of typically six to eight percent can be completely recovered by heating the nickel-titanium alloys (Nitinol) above the austenite finish temperature. The transformation temperatures can be altered by changing the composition of the alloy. In addition, when 
Nitinol is heated, the Young's modulus increases three to four times and the yielding strength also increases approximately ten times ${ }^{3-6}$.

The materials, referred to in this study as SMA fiber-reinforced hybrid composites, are conventional advanced composite materials (such as graphite-epoxy) that contain embedded SMA fibers having the same direction as the graphite fibers. The memory effect of the SMA fibers may be put to use by applying an initial elongation to them during fabrication of the laminate. Thus, once the laminate is heated above $T_{s}$, the SMA fibers will try to recover the initial strain and induce tensile inplane forces in the laminate. Since the expected aerodynamic heating on the skin panels of a HSCT during supersonic cruise will generate panel temperatures well above the austenite finish temperature, SMA will be ideal for such an application. The overall stiffness of the hybrid composite panel will be increased due to: 1) the increase of the Young's modulus of the SMA fibers by a factor of three or four, and 2) the internal tensile inplane forces induced in the panel from the recovery of initial strains of the SMA fibers. Therefore, the root-mean-square (RMS) maximum deflection and RMS maximum strain, due to random pressure loading, will be reduced at elevated temperatures as compared to composite panels without embedded SMA fibers. This has been demonstrated in a preliminary investigation on passive control of random response of SMA hybrid composite plates using the classic analytical continuum method ${ }^{7}$.

Shape memory alloys have been applied as actuators for active control of buckling of beams ${ }^{8}$ and shape control of beams ${ }^{9}$. It is also being studied for use in active vibration control of beams ${ }^{10,11}$ and large space structures ${ }^{12}$. Active vibration control of flexible linkage mechanisms using SMA fiber-reinforced composites has been investigated by Venkatesh et al. ${ }^{13}$. Acoustic transmission and radiation control through the use of SMA fibers in a hybrid composite was presented by Liang and Anders ${ }^{14,15}$. In all of these investigations $^{8-15}$, the SMA fibers or strips were heated by applying an electrical current with some control device. Since it was assumed that the composite would not be appreciably heated, thermal expansion effects were not included. However, the heat source for the present study is assumed to be due to aerodynamic heating. Therefore no control device is needed, but thermal expansion effects are important since the entire laminate is heated and thermal buckling and postbuckling considerations may be necessary.

Consider a thin composite lamina, for example graphite-epoxy, having arbitrary orientation angle $\theta$ and embedded SMA fibers in the same direction as the graphite fibers, Figure 1. The 1 and 2 directions, or principal material directions, are parallel and perpendicular to the fiber direction respectively. The constitutive relations for such a lamina can be derived following the engineering approach found in many composite materials texts. A derivation may be found in the appendix and the resulting constitutive relations, in principal material coordinates, are given by Eqs. (A17) and (A18). For a general k-th layer with an orientation angle $\theta$, the stress-strain relations, Eqs. (A17) and (A18), become

$$
\begin{aligned}
\{\sigma\}_{k} & =\left\{\begin{array}{c}
\sigma_{x} \\
\sigma_{y} \\
\tau_{x y}
\end{array}\right\}_{k}=\left[\bar{Q}^{*}\right]_{k}\left\{\begin{array}{c}
\epsilon_{x} \\
\epsilon_{y} \\
\gamma_{x y}
\end{array}\right\}+\left\{\begin{array}{c}
\sigma_{x}^{*} \\
\sigma_{y}^{*} \\
\tau_{x y}^{*}
\end{array}\right\}_{k} v_{s k} \\
& -\left([\bar{Q}]_{m}\left\{\begin{array}{c}
\alpha_{x} \\
\alpha_{y} \\
\alpha_{x y}
\end{array}\right\}_{m} v_{m}\right)_{k} \Delta, \quad T>T_{s} \\
\{\sigma\}_{k}= & {\left.\left[\bar{Q}^{*}\right]_{k}\left(\left\{\begin{array}{c}
\epsilon_{x} \\
\epsilon_{y} \\
\gamma_{x y}
\end{array}\right\}-\left\{\begin{array}{c}
\alpha_{x} \\
\alpha_{y} \\
\alpha_{x y}
\end{array}\right\}_{k}\right) \Delta T\right), T<T_{s} }
\end{aligned}
$$

The resultant force and moment vectors of the SMA fiber-reinforced hybrid composite plate are defined as

$$
\{N, M\}=\int_{-h / 2}^{h / 2}\{\sigma\}_{k}(1, z) d z
$$

$\mathrm{Or}^{\mathrm{N}}$

$$
\left\{\begin{array}{c}
N \\
M
\end{array}\right\}=\left[\begin{array}{ll}
A^{*} & B^{*} \\
B^{*} & D^{*}
\end{array}\right]\left\{\begin{array}{c}
\epsilon^{o} \\
\kappa
\end{array}\right\}+\left\{\begin{array}{c}
N_{\sigma}^{*} \\
M_{\sigma}^{*}
\end{array}\right\}-\left\{\begin{array}{c}
N_{\Delta T} \\
M_{\Delta T}
\end{array}\right\}
$$

where the laminate stiffness $\left[A^{*}\right], \quad\left[B^{*}\right]$ and $\left[D^{*}\right]$ and the recovery inplane force and moment vectors $\left\{N_{\sigma}^{*}\right\}$ and $\left\{M_{\sigma}^{*}\right\}$ are all temperature dependent. The inplane strain and curvature vectors are defined from the von Karman strain-displacement relations

$$
\begin{aligned}
\left\{\begin{array}{c}
\epsilon_{x} \\
\epsilon_{y} \\
\gamma_{x y}
\end{array}\right\} & =\left\{\epsilon^{o}\right\}+z\{\kappa\} \\
& =\left\{\epsilon_{m}^{o}\right\}+\left\{\epsilon_{b}^{o}\right\}+z\{\kappa\} \\
& =\left\{\begin{array}{c}
u, x \\
v, y \\
u, y+v, x
\end{array}\right\}+\frac{1}{2}\left\{\begin{array}{c}
w,{ }_{x}^{2} \\
w, y \\
2 w, x w, y
\end{array}\right\}-z\left\{\begin{array}{c}
w, x x \\
w, y y \\
2 w, x y
\end{array}\right\}
\end{aligned}
$$

\section{Finite Element Formulation}

A limited number of investigations on the structural response of panels subjected to combined acoustic and thermal loads exist in the literature. Seide and 
Adami ${ }^{16}$ were the first who studied large deflection random response of a thermally buckled beam. The well-known classic Woinowsky-Krieger large amplitude beam vibration equation was used. Galerkin's method and time domain numerical simulation were then applied to obtain the random response. More recently, the Galerkin/numerical simulation approach was applied to simply supported metal and orthotropic composite rectangular plates by Vaicaitis et al. ${ }^{17,18}$. The classic von Karman large-deflection plate equations including uniform temperature and orthotropic property effects were employed. Lee ${ }^{19}$ extended that work to include nonuniform temperature distributions. He studied isotropic rectangular plates with either simply supported or clamped edges. The Galerkin/equivalent linearization method ${ }^{20}$ was used. The classic continuum approaches thus have been limited to simple beams ${ }^{16}$ and isotropic or orthotropic rectangular plates ${ }^{17-19}$.

For over three decades, the finite element method has been the predominant approach for structural mechanics of complex structural geometry. However, there are few studies where the random response of structures subjected to combined acoustic and thermal loads is involved. Locke and Mei ${ }^{21,22}$ extended the finite element method for the first time to beam and plate structures under combined thermal and acoustic loads. The thermal load considered was a steadystate temperature distribution $\Delta T(x, y)$. Chen and $\mathrm{Mei}^{23}$ recently refined that finite element formulation for nonlinear random response of structures by considering the acoustic pressure and thermal load to be applied simultaneously.

The governing equations of motion can be derived for a SMA fiber-reinforced hybrid composite panel subjected to combined thermal and acoustic loads through the use of a variational principle. These equations may be written in the form

$$
\begin{aligned}
& {\left[\begin{array}{cc}
M_{b} & 0 \\
0 & M_{m}
\end{array}\right]\left\{\begin{array}{c}
\ddot{W}_{b} \\
\ddot{W}_{m}
\end{array}\right\}+\left(\left[\begin{array}{cc}
K_{b} & K_{B} \\
K_{B}^{T} & K_{m}
\end{array}\right]+\left[\begin{array}{cc}
K_{\sigma}^{*} & 0 \\
0 & 0
\end{array}\right]\right.} \\
& -\left[\begin{array}{cc}
K_{N \Delta T} & 0 \\
0 & 0
\end{array}\right]+\frac{1}{2}\left[\begin{array}{cc}
N 1_{N m}+N 1_{N B} & N 1_{b m} \\
N 1_{m b} & 0
\end{array}\right] \\
& \left.+\frac{1}{3}\left[\begin{array}{cc}
N 2_{b} & 0 \\
0 & 0
\end{array}\right]\right)\left\{\begin{array}{c}
W_{b} \\
W_{m}
\end{array}\right\}=\left\{\begin{array}{c}
P_{b}(t) \\
0
\end{array}\right\}+\left\{\begin{array}{c}
P_{b \Delta T} \\
P_{m \Delta T}
\end{array}\right\} \\
& -\left\{\begin{array}{c}
P_{b \sigma}^{*} \\
P_{m \sigma}^{*}
\end{array}\right\}
\end{aligned}
$$

or

$$
\begin{aligned}
& {[M]\{\ddot{W}\}+\left([K]+\left[K_{\sigma}^{*}\right]-\left[K_{N \Delta T}\right]\right.} \\
& \left.+\frac{1}{2}[N 1]+\frac{1}{3}[N 2]\right)\{W\}=\{P(t)\} \\
& +\left\{P_{\Delta T}\right\}-\left\{P_{\sigma}^{*}\right\}
\end{aligned}
$$

where $[M]$ and $[K]$ are the system mass and stiffness matrices; $\left[K_{\sigma}^{*}\right]$ and $\left[K_{N \Delta T}\right]$ are the geometric stiffness matrices due to the recovery stress $\sigma_{r}^{*}$ (or $\left.\left\{N_{\sigma}^{*}\right\}\right)$ and thermal inplane force vector $\left\{N_{\Delta T}\right\}$, respectively; [N1] and [N2] are the first and second order nonlinear stiffness matrices which depend linearly and quadratically upon displacement $\{W\}$, respectively; $\{P(t)\}$ is the random acoustic excitation load vector, $\left\{P_{\Delta T}\right\}$ is the thermal load vector, and $\left\{P_{\sigma}^{*}\right\}$ is the SMA recovery force vector. The subscripts $b$ and $m$ denote bending and membrane components, respectively; and the subscripts $B, N m$ and $N B$ indicate that the corresponding stiffness matrix is due to the laminate stiffness $[B]$, membrane forces $\left\{N_{m}\right\}\left(=[A]\left\{\epsilon_{m}^{o}\right\}\right)$ and $\left\{N_{B}\right\}(=[B]\{\kappa\})$, respectively. The stiffness matrices $[K],[N 1]$ and $[N 2]$ and the recovery force vector $\left\{P_{\sigma}^{*}\right\}$ are also temperature dependent. Detailed derivations of the equations of motion and expressions for the element matrices and load vectors are referred to reference 21 . The solution procedures developed in references 21-23 are to be extended to obtain the critical buckling temperature, thermal postbuckling deflection, and linear RMS maximum deflection of SMA hybrid composite panels.

\section{Solution Procedures}

Four different types of analyses are required for sonic fatigue design of SMA hybrid composite panels. They are: (1) thermal buckling analysis, (2) random vibration analysis, (3) thermal postbuckling analysis, and (4) random vibration analysis of thermally buckled panels. Each analysis is discussed briefly in the following.

\section{Thermal Buckling Analysis}

As the first step, a thermal buckling analysis is carried out to determine the critical buckling temperature $T_{c r}=\Delta T_{c r}+T_{o}$, where $T_{o}$ is the reference or ambient temperature. Equations governing the thermal buckling are obtained by neglecting the inertia, nonlinear, bending-membrane coupling, and bending force terms from the incremental form of Eq. (5) as

$$
\left(\left[K_{b}\right]+\left[K_{\sigma}^{*}\right]-\left[K_{N \Delta T}\right]+\left[N 1_{N m}\right]\right)\left\{\Delta W_{b}\right\}=0
$$

and

$$
\left[K_{m}\right]\left\{W_{m}\right\}=\left\{P_{m \Delta T}\right\}-\left\{P_{m \sigma}^{*}\right\}
$$

The first-order nonlinear stiffness matrix $\left[N 1_{N m}\right]$ is linearly dependent upon the inplane displacement $\left\{W_{m}\right\}$ which can be expressed at a given $\Delta T$ as

$$
\begin{aligned}
\left\{W_{m}\right\} & =\left[K_{m}\right]^{-1}\left(\left\{P_{m \Delta T}\right\}-\left\{P_{m \sigma}^{*}\right\}\right) \\
& =\left\{W_{m}\right\}_{1}-\left\{W_{m}\right\}_{o}
\end{aligned}
$$


where $\left\{W_{m}\right\}_{1}=\left[K_{m}\right]^{-1}\left\{P_{m \Delta T}\right\}$ is linearly dependent upon $\Delta T$ and $\left\{W_{m}\right\}_{o}=\left[K_{m}\right]^{-1}\left\{P_{m \sigma}^{*}\right\}$ is a constant vector. Thus, the matrix $\left[N 1_{N m}\right]$ is the sum of two matrices: the first matrix $\left[N 1_{N m}\right]_{1}$ is evaluated with $\left\{W_{m}\right\}_{1}$ and the second matrix $\left[N 1_{N m}\right]_{0}$ is evaluated with $\left\{W_{m}\right\}_{0}$ as

$$
\left[N 1_{N m}\right]=\left[N 1_{N m}\right]_{1}-\left[N 1_{N m}\right]_{o}
$$

The matrices $\left[K_{N \Delta T}\right]$ and $\left[N 1_{N m}\right]_{1}$ are linearly dependent upon $\Delta T$. Therefore, Eq. (7) can be expressed as an eigen-problem

$$
\begin{gathered}
\left(\left[K_{b}\right]+\left[K_{\sigma}^{*}\right]-\left[N 1_{N m}\right]_{o}\right)\{\phi\}=\lambda\left(\left[K_{N \Delta T}\right]\right. \\
\left.-\left[N 1_{N m}\right]_{1}\right)\{\phi\}
\end{gathered}
$$

The critical temperature change corresponds to the lowest value of $\lambda$ and is given by $\Delta T_{c r}=$ $\lambda \Delta T$, and $\{\phi\}$ is the corresponding buckling mode. Since the matrices $\left[\mathrm{K}_{\mathrm{b}}\right],\left[\mathrm{K}_{\sigma}^{*}\right],\left[N 1_{N m}\right]_{0}$ and $\left[N 1_{N m}\right]_{1}$ in Eq. (11) are all temperature dependent, an iterative scheme is implemented to achieve convergence for $\Delta \mathrm{T}_{\text {cr }}(\lambda=1.000 \pm .001)$.

\section{Random Vibration Analysis}

Once the critical buckling temperature is determined, the next step will depend on the panel temperature for the particular application of concern. For panel temperatures greater than the critical temperature $\left(T>T_{c r}\right)$, a thermal postbuckling analysis has to be performed, followed by a random vibration analysis of the thermally buckled structure.

For panel temperatures less than the critical temperature $\left(T<T_{c r}\right)$, the panel is flat. A random vibration analysis including the thermal and SMA effects is performed. The response results include frequencies and modes of vibration, RMS maximum deflection, and power spectral density (PSD) functions of maximum deflection. The equations of motion for a SMA hybrid composite panel subjected to a combined acoustic excitation and thermal load can be obtained from Eq. (6) as

$$
[M]\{\ddot{W}\}+[\bar{K}]\{W\}=\{P(t)\}
$$

where

$$
[\bar{K}]=[K]+\left[K_{\sigma}^{*}\right]-\left[K_{N \triangle T}\right]+[N 1]
$$

where the only nonzero entries in [N1] are due to $\left[\mathrm{N}_{\mathrm{Nm}}\right]$ resulting from the inplane deflection, Eq. (9).

The natural frequencies $\omega_{r}$ and mode shapes $\left\{\phi_{r}\right\}$ of vibration are obtained from the eigenvalue problem

$$
\omega_{r}^{2}[M]\left\{\phi_{r}\right\}=[\bar{K}]\left\{\phi_{r}\right\}
$$

A set of uncoupled modal equations with reduced degrees-of-freedom can thus be obtained from Eq. (12) as

$$
\ddot{q}_{r}+2 \zeta_{r} \omega_{r} \dot{q}_{r}+\omega_{r}^{2} q_{r}=f_{r}, \quad r=1,2, \ldots, N
$$

with the truncated modal transformation

$$
\{W\}=\sum_{r=1}^{N}\left\{\phi_{r}\right\} q_{r}=[\phi]\{q\}
$$

In Eq. (15), a modal damping term has been included where $\zeta_{r}$ is the modal damping ratio and the modal mass and force are

$$
\begin{gathered}
m_{r}=\left\{\phi_{r}\right\}^{T}\left[M_{b}\right]\left\{\phi_{r}\right\} \\
f_{r}=\left\{\phi_{r}\right\}^{T}\left\{P_{b}(t)\right\} / m_{r}
\end{gathered}
$$

The random modal response can be easily determined from Eq. (15) for the case of a spatially uniform white noise random load $p(t)$ with spectral density $S_{p}$ or $G_{p}$ as

$$
\begin{aligned}
E\left[q_{r} q_{s}\right] & =\int_{-\infty}^{\infty} S_{p}(\omega) H_{r}(\omega) H_{s}(-\omega) d \omega \\
& =\int_{0}^{\infty} G_{p}(f) H_{r}(f) H_{s}(-f) d f
\end{aligned}
$$

with $G_{p}(f)=4 \pi S_{p}(\omega)$ for $\omega=2 \pi f(f \geq 0)$ and

$$
H_{r}(\omega)=\frac{1}{\omega_{r}^{2}-\omega^{2}+2 i \zeta_{r} \omega_{r} \omega}
$$

The cross-correlation of modal amplitudes from Eq. (18) is

$$
\begin{gathered}
E\left[q_{r} q_{s}\right]=\frac{G_{p}(f)\left(\zeta_{r} \omega_{r}+\zeta_{s} \omega_{s}\right)}{m_{r} m_{s}\left\{\left(\omega_{r}^{2}-w_{s}^{2}\right)^{2}+4\left[\zeta_{r} \zeta_{s} \omega_{r} \omega_{s}\left(\omega_{r}^{2}+\omega_{s}^{2}\right)\right.\right.} \\
\left.\left.+\left(\zeta_{r}^{2}+\zeta_{s}^{2}\right) \omega_{r}^{2} \omega_{s}^{2}\right]\right\}
\end{gathered}
$$

and the correlation matrix of the system nodal amplitude becomes

$$
E\left[\{W\}\{W\}^{T}\right]=[\phi] E\left[\{q\}\{q\}^{T}\right][\phi]^{T}
$$

\section{Thermal Postbuckling Analysis}

For panel temperatures greater than the critical temperature $\left(T>T_{c r}\right)$, postbuckling analysis is performed to determine the thermal deflection. The governing equations for thermal postbuckling can be obtained from Eq. (6) as

$$
\begin{gathered}
\left([K]+\left[K_{\sigma}^{*}\right]-\left[K_{N \Delta T}\right]+\frac{1}{2}[N 1]+\frac{1}{3}[N 2]\right)\{W\} \\
=\left\{P_{\Delta T}\right\}-\left\{P_{\sigma}^{*}\right\}
\end{gathered}
$$


One effective approach involves using the NewtonRaphson iterative method for solving time-independent nonlinear problems. Thus, for the $\mathbf{j}$-th iteration the incremental form of Eq. (22) can be written as

$$
\left[K_{\mathrm{tan}}\right]_{j}\{\Delta W\}_{j+1}=\{\Delta P\}_{j}
$$

Then $\left[K_{\tan }\right]_{j+1}$ and $\{\Delta P\}_{j+1}$ are updated by using

$$
\left\{W_{\triangle T}\right\}_{j+1}=\left\{W_{\triangle T}\right\}_{j}+\{\Delta W\}_{j+1}
$$

The solution process seeks to reduce the imbalance load vector $\{\Delta P\}$, and consequently $\{\Delta W\}$, to a specified small quantity. The tangent stiffness matrix and the imbalance load vector are

$\left[K_{\mathrm{tan}}\right]_{j}=[K]+\left[K_{\sigma}^{*}\right]-\left[K_{N \Delta T}\right]+\left[N 1_{\triangle T}\right]_{j}+\left[N 2_{\triangle T}\right]_{j}$

and

$$
\begin{aligned}
\{\Delta P\}_{j} & =\left\{P_{\Delta T}\right\}-\left\{P_{\sigma}^{*}\right\}-\left([K]+\left[K_{\sigma}^{*}\right]-\left[K_{N \Delta T}\right]\right. \\
& \left.+\frac{1}{2}\left[N 1_{\triangle T}\right]_{j}+\frac{1}{3}\left[N 2_{\triangle T}\right]_{j}\right)\left\{W_{\triangle T}\right\}_{j}
\end{aligned}
$$

where the subscript $\triangle T$ denotes that the nonlinear stiffness matrix is evaluated using $\left\{W_{\triangle T}\right\}$. The linear buckling mode shape, Eqs. (9) and (11), normalized by a scale factor, is usually taken to be the initial trial solution for Eq. (23).

\section{Random Vibration Analysis of Buckled Panels}

The random vibration analysis of a thermally buckled panel is performed to determine the frequencies and modes of vibration about the buckled position, the RMS maximum deflection, and the PSD functions. The equations of motion for a thermally buckled SMA hybrid composite panel can be obtained from Eq. (6) and ref. 24 as

$$
\begin{aligned}
{[M]\{\ddot{W}\} } & +\left([K]+\left[K_{\sigma}^{*}\right]-\left[K_{N \Delta T}\right]+\left[N 1_{\triangle T}\right]\right. \\
& +[N 2 \triangle T])\{W\}=\{P(t)\}
\end{aligned}
$$

where the subscript $\triangle T$ denotes that the corresponding stiffness matrix is evaluated using the thermally buckled deflection $\left\{W_{\triangle T}\right\}$. The influences of recovery stress $\sigma_{r}^{*}$, thermal force $\left\{N_{\Delta T}\right\}$ and thermal postbuckling deflection are given by the presence of $\left[K_{\sigma}^{*}\right],\left[K_{N \Delta T}\right],\left[N 1_{\triangle T}\right]$ and $\left[N 2_{\triangle T}\right]$ in Eq. (27). An examination of Eqs. (25) and (27) reveals a novel approach of the present finite element formulation. That is, the stiffness matrices in Eq. (27) need not to be computed and assembled. The sum of those stiffness matrices is exactly the converged tangent stiffness matrix in thermal postbuckling analysis. Thus, Eq. (27) is simply

$$
[M]\{\ddot{W}\}+\left[K_{\text {tan }}\right]\{W\}=\{P(t)\}
$$

The natural frequencies and mode shapes of vibration about the thermally buckled position $\left\{W_{\triangle T}\right\}$ are determined from

$$
\omega_{s}^{2}[M]\left\{\phi_{s}\right\}=\left[K_{\text {tan }}\right]\left\{\phi_{s}\right\}
$$

Equation (27) thus becomes a set of uncoupled modal equations

$$
\ddot{q}_{s}+2 \zeta_{s} \omega_{s} \dot{q}_{s}+\omega_{s}^{2} q_{s}=f_{s}, \quad s=1,2, \ldots, N
$$

with the truncated modal transformation

$$
\{W\}=\sum_{s=1}^{N}\left\{\phi_{s}\right\} q_{s}=[\phi]\{q\}
$$

The determination of the random response follows from Eqs. (17)-(21).

\section{$\underline{\text { Results and Discussion }}$}

The results shown in this paper were generated for a SMA hybrid composite laminate, where the graphiteepoxy composite was treated as the matrix. The following material properties were used in the analysis:

$\begin{array}{ll}\begin{array}{l}\text { Graphite-Epoxy } \\ \mathrm{E}_{1}\end{array} & 155 \mathrm{GPa}(22.5 \mathrm{Msi}) \\ \mathrm{E}_{2} & 8.07(1.17) \\ \mathrm{G}_{12} & 4.55(0.66) \\ \nu_{12} & 0.22 \\ \rho & 1550.07 \mathrm{Kg} / \mathrm{m}^{3}\left(0.1458 \times 10^{-3} \mathrm{lb}-\mathrm{s}^{2} / \mathrm{in} .^{4}\right) \\ \alpha_{1} & -0.07 \times 10^{-6} /{ }^{\circ} \mathrm{C}\left(-0.04 \times 10^{-6} /{ }^{\circ} \mathrm{F}\right) \\ \alpha_{2} & 30.1 \times 10^{-6} \quad\left(16.7 \times 10^{-6}\right)\end{array}$

SMA-Nitinol

$\mathrm{T}_{\mathrm{s}} \quad 37.78^{\circ} \mathrm{C}\left(100^{\circ} \mathrm{F}\right)$

$\mathrm{T}_{\mathrm{f}} \quad 62.78(145)$

E From Fig. A3 


$$
\begin{aligned}
& \text { G } \quad 24.86 \quad(3.604) \text { for } \mathrm{T}<\mathrm{T}_{\mathrm{s}} \\
& 25.6 \quad(3.712) \text { for } \mathrm{T}>\mathrm{T}_{\mathrm{f}} \\
& \nu \quad 0.3 \\
& \rho \quad 6450 \mathrm{Kg} / \mathrm{m}^{3}\left(0.6067 \times 10^{-3}{\left.\mathrm{lb}-\mathrm{s}^{2} / \mathrm{in} .^{4}\right)}^{4}\right. \\
& \alpha \quad 10.26 \times 10^{-6} /{ }^{\circ} \mathrm{C}\left(5.7 \times 10^{-6} /{ }^{\circ} \mathrm{F}\right)
\end{aligned}
$$

Table 1 presents the results for a simply supported rectangular laminate with a graphite-epoxy matrix. The dimensions of the laminate are $15 \times 12 \times 0.048 \mathrm{in}$. and the layup is symmetric $(0 / 45 /-45 / 90)_{\mathrm{s}}$. Results are presented for a laminate with no SMA fibers and for three SMA initial strain values $(3 \%, 4 \%$ and $5 \%$ ) for each nonzero SMA volume fraction. The headings indicate SMA volume fraction, SMA fiber initial strain, two critical temperature changes, normalized maximum thermal deflection, and normalized maximum RMS deflection.

Note that when SMA fibers are present in the laminate, there are at least two critical buckling temperatures (ambient temperature $70^{\circ} \mathrm{F}$ ). One corresponds to temperatures less than the austenite start temperature $T_{s}$, and the other corresponds to temperatures greater than the austenite finish temperature $T_{f}$. It can be seen that the addition of SMA fibers slightly reduces the critical buckling temperature below $T_{s}$. This is caused by the relatively low modulus of the SMA fibers in relation to the graphite epoxy matrix they are replacing. However, with the addition of only $10 \%$ SMA fibers at $3 \%$ initial strain, the critical buckling temperature above $T_{f}$ is increased drastically. Some of the reported critical buckling temperatures are unrealistic for a graphite-epoxy matrix since epoxy composites are generally considered to have a maximum service temperature of around $300^{\circ} \mathrm{F}$. However, these results are qualitatively indicative of the shape memory effect and allude to the potential use of SMA fibers in high temperature matrices such as metals, carbon-carbon, etc.

It can be seen that the thermal deflection $W_{\Delta \mathrm{T}} / \mathrm{h}$ is attenuated in all cases and even eliminated in most. The themal deflection is eliminated for the cases with $10 \%$ SMA fibers at $5 \%$ initial strain and all greater volume fractions since the critical buckling temperature has been shifted well above the panel temperature $\left(300^{\circ} \mathrm{F}\right)$. This effect is beneficial due to the fact that the optimal structural configuration may be maintained and the possibility of snap-through motions is eliminated.

Although the dynamic response $W_{\max } / \mathrm{h}$ of panels with some of the lower SMA volume fractions and initial strains is actually greater than that for the panel without SMA fibers, significant dynamic response at- tenuation may be achieved with SMA volume fractions of thirty percent or more. The increase in the dynamic response, observed for some of the cases, is due to the relatively low modulus of the Nitinol alloy, even at high temperatures and another factor which will be discussed subsequently. Finally, the case of $10 \%$ SMA and $4 \%$ initial strain exemplifies the importance of choosing the SMA parameters carefully, as indicated by the excessively large RMS maximum deflection. This particular choice results in a critical buckling temperature of $295.06^{\circ} \mathrm{F}$, which is within $2 \%$ of the panel temperature $\left(300^{\circ} \mathrm{F}\right)$. Thus, the panel is very compliant since it is close to buckling.

The variation of the RMS ( $\left.W_{\max } / h\right)$ with sound spectrum level (SSL) is shown in Figure 2 for four cases: no SMA fibers at $70^{\circ} \mathrm{F}, 10 \%$ SMA fibers and $5 \%$ initial strain at $300^{\circ} \mathrm{F}$, no SMA at $300^{\circ} \mathrm{F}$, and $30 \%$ SMA and $5 \%$ initial strain at $300^{\circ} \mathrm{F}$. Note that the panel without SMA fibers at $300^{\circ} \mathrm{F}$ exhibits significantly reduced dynamic response because of the additional stiffness due to the fact that the panel is thermally buckled. Adding $10 \%$ SMA fibers to the $300^{\circ} \mathrm{F}$ panel increases the dynamic response since the recovery forces induced are not sufficient to overcome the modulus deficiency of the SMA fibers and the loss of stiffness due to the fact that the panel is no longer thermally buckled. Finally, addition of $30 \%$ SMA fibers provides ample recovery forces to significantly reduce the dynamic response, even more than the thermally buckled case.

The variation of the RMS ( $W_{\max } / h$ ) with temperature for a laminate with no SMA fibers in comparison to one with $30 \%$ SMA and $5 \%$ initial strain is shown in Figure 3 . Note that they both exhibit a peak, albeit beyond the linear range $\left(W_{\max } / \mathrm{h}<1\right)$, at their respective low critical temperatures due to extremely low stiffness at this point of instability. In addition, the response of the laminate with SMA fibers has a peak at $100^{\circ} \mathrm{F}$ since the SMA modulus has a local minimum at that temperature.

The power spectral density of the maximum deflection at $100 \mathrm{~dB}$ is shown in Figures $4-6$ for three cases: no SMA at $70^{\circ} \mathrm{F} ; 30 \%$ SMA and $5 \%$ initial strain at $70^{\circ} \mathrm{F}$; and $30 \%$ SMA and $5 \%$ initial strain at $300^{\circ} \mathrm{F}$, respectively. At low temperature, the fundamental frequency of the panel with SMA fibers is lower than that for the panel with no SMA since the SMA reinforced panel is less stiff at low temperatures and more massive. At $300^{\circ} \mathrm{F}$, the modes of the SMA fiber-reinforced panel exhibit significant amplitude reduction and have shifted to much higher frequencies. 


\section{Conclusions}

The finite element method has been successfully implemented to analyze the thermal buckling, thermal postbuckling, and random response of SMA fiberreinforced hybrid composite panels. The constitutive relations, including the thermal and shape recovery effects, have been derived and used in the finite element formulation to model the mechanics of a SMA/graphite-epoxy panel.

It was found that panels with SMA fiber reinforcement have at least two critical buckling temperatures; one below the austenite start temperature $T_{s}$, and one above the austenite finish temperature $T_{f}$. The extremely high critical buckling temperatures predicted for SMA volume fractions greater than $10 \%$ indicate the possible suitability of SMA fibers in high temerature composites.

The thermal postbuckling deflection can be totally eliminated, for panel temperatures greater than $T_{f}$, with as little as $10 \%$ SMA fibers. The property could be useful in practical applications by maintaining optimal aerodynamic configuration and eliminating snap-through motions.

The RMS maximum deflection actually increases for some SMA volume fractions because of two factors: the modulus of the SMA fibers is relatively low, even at high temperatures; and the recovery forces induced by small volume fractions of SMA fibers is sufficient to prevent thermal buckling (for $\mathrm{T}_{\mathrm{f}}<\mathrm{T}<\mathrm{T}_{\mathrm{cr}}$ ) but insufficient to overcome the loss of the buckling stiffness. However, significant reductions in the dynamic response are realizable with SMA volume fractions of $30 \%$ or more.

\section{Acknowledgments}

The second and third authors would like to acknowledge the partial support by NAG1-1358, NASA Langley Research Center and F33615-91-C-3205, AF Wright Laboratory.

\section{References}

1. Mixson, J. S. "Overview of Acoustic Fatigue Activities at NASA Langley Research Center," AFWAL-TR-88-3034. Wright-Patterson AFB, July 1988 , pp. 573-591.

2. Dehart, D. and Griffin, S., "Astronautical Laboratory Intelligent Structure/Skins Overview," Proceedings of the First Joint US/Japan Conference on Adaptive Structures, Maui, Hawaii, November 13-15, 1990, pp. 3-10.
3. Cross, W. B., Kariotis, A. H. and Stimler, F. J., "Nitinol Characterization Study," NASA CR1433, 1969.

4. Jackson, C. M., Wagner, H. J. and Wasilewski, R. J., "55-Nitinol The Alloy with a Memory: Its Physical Metallurgy, Properties, and Applications," NASA SP-5110, 1972.

5. Rogers, C. A., Liang, C. and Jia, J., "Behavior of Shape Memory Alloy Reinforced Composite Plates - Part 1: Model Formulation and Control Concepts," Proceedings of the 30th Structures, Structural Dynamics and Materials Conference, Mobile, AL, April 3-5, 1989, pp. 2011-2017.

6. Jia, J. and Rogers, C. A., "Formulation of a Mechanical Model of Composites with Embedded SMA Actuators," Proceedings of the 8th Biennial Conference on Failure Prevention and Reliability, Montreal, Canada, September, 17-21, 1989, pp. $203-210$.

7. Pates III, C. S., Zhong, Z.. W. and Mei, C., "Passive Control of Random Response of Shape Memory Alloy Fiber-Reinforced Composite Plates," To be Presented at the 5th International Conference on Recent Advances in Structural Dynamics, Institute of Sound and Vibration, University of Southampton, July 18-21, 1994.

8. Baz, A. and Tampe, L., "Active Control of Buckling of Flexible Beams," Proceedings of the 8th Biennial Conference on Failure Prevention and Reliability, Montreal, Canada, September 17-21, 1989, pp. 211-218.

9. Chaudhry, Z. and Rogers, C. A., "Bending and Shape Control of Beams Using SMA Actuators," Journal of Intelligent Systems and Structures, Vol. 2, 1991, pp. 581-602.

10. Baz, A., Iman, K. and McCoy, J., "Active Vibration Control of Flexible Beams Using Shape Memory Actuators," Journal of Sound and Vibration, Vol. 140, 1990, pp. 437-456.

11. Srinivasan, A. V., Cutts, D. G. and Schetky, L. M., "Thermal and Mechanical Consideration in Using Shape Memory Alloys to Control Vibrations in Flexible Structures," Metallurgical Transactions A, Vol. 22A, 1991, pp. 623-627.

12. Maclean, B. J., Patterson, G. J. and Misra, M. S., "Modeling of a Shape Memory Integrated Actuator for Vibration Control of Large Space Structures," Journal of Intelligent Materials Systems and Structures, Vol. 2, 1991, pp. 72-94. 
13. Venkatesh, A., Hilborn, J., Bidaux, J. E. and Gotthardt, R., "Active Vibration Control of Flexible Linkage Mechanisms Using Shape Memory Alloy Fiber-Reinforced Composites," The 1st European Conference on Smart Structures and Materials, Glasgow, UK, May 12-14, 1992, pp. 185-188.

14. Liang, C., Rogers, C. A. and Fuller, C. R., "Acoustic Transmission and Radiation Analysis of Adaptive Shape Memory Alloy Reinforced Laminated Plates," Journal of Sound and Vibration, Vol. 145, 1991, pp. 23-41.

15. Anders, W. S., Rogers, C. A. and Fuller, C. R., "Vibration and Low Frequency Acoustic Analysis of Piecewise-Activated Adaptive Composite Panels," Journal of Composite Materials, Vol. 26, 1992, pp. 103-120.

16. Seide, P. and Adami, C., "Dynamic Stability of Beams in a Combined Thermal-Acoustic Environment," AFFWAL-TR-83-3027, Wright-Patterson AFB, October 1983.

17. Vaicaitis, R. and Arnold, R. R., "Nonlinear Response and Fatigue of Surface Panels by the Time Domain Monte Carlo Approach," WRDC-TR-903081, Wright-Patterson AFB, May 1992.

18. Vaicaitis, R. and Kavallieratos, P. A., "Nonlinear Response of Composite Panels to Random Excitation," Proceedings of the 34th Structures, Structural Dynamics and Materials Conference, LaJolla, CA, April 19-22, 1993, pp. 1041-1049.

19. Lee, J., "Large-Amplitude Plate Vibration in an Elevated Thermal Environment," WL-TR-923049, Wright-Patterson AFB, June 1992.

20. Roberts, J. B. and Spanos, P. D., Random Vibration and Statistical Linearization, John Wiley, New York, 1990.

21. Locke, J. E., “A Finite Element Formulation for the Large Deflection Random Response of Thermally Buckled Structures," Ph.D. Dissertation, Old Dominion University, Norfolk, VA, July 1988.

22. Locke, J. E. and Mei, C., "A Finite Element Formulation for the Large Deflection Random Response of Thermally Buckled Beams," AIAA Journal, Vol. 28, 1990, pp. 2125-2131.

23. Chen, R. and Mei, C., "Finite Element Nonlinear Random Response of Beams to Acoustic and Thermal Loads Applied Simultaneously," Proceedings of the 34th Structures, Structural Dynamics and Materials Conference, LaJolla, CA, April 19-22, 1993, pp. 1050-1057.

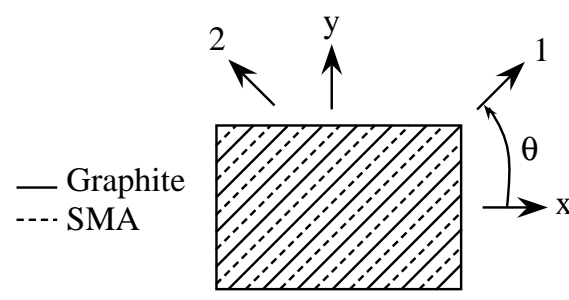

Figure 1: SMA fiber-reiforced hybrid compostite lamina.

\begin{tabular}{|c|c|c|c|c|c|}
\hline $\mathrm{v}_{\mathrm{S}}$ & $\epsilon_{\mathrm{r}}$ & $\begin{array}{l}\Delta \mathrm{T}_{\mathrm{cr}} \\
\mathrm{T}<\mathrm{T}_{\mathrm{s}}\end{array}$ & $\begin{array}{l}\Delta \mathrm{T}_{\mathrm{cr}} \\
\mathrm{T}>\mathrm{T}_{\mathrm{f}}\end{array}$ & $\begin{array}{c}\mathrm{W}_{\Delta_{\mathrm{T}}} / \mathrm{h} \\
\mathrm{at} \\
300^{\circ} \mathrm{F}\end{array}$ & $\begin{array}{l}\text { RMS } W_{\max } / \mathrm{h} \\
\text { at } 300^{\circ} \mathrm{F} \\
\mathrm{SSL}=100 \mathrm{~dB}\end{array}$ \\
\hline $0 \%$ & - & 13.73 & 13.73 & 2.7732 & 0.0616 \\
\hline \multirow{3}{*}{$10 \%$} & $3 \%$ & \multirow{3}{*}{11.93} & 192.25 & 1.1605 & 0.2181 \\
\hline & $4 \%$ & & 225.06 & 0.4308 & 1.0113 \\
\hline & $5 \%$ & & 277.38 & - & 0.3173 \\
\hline \multirow{3}{*}{$20 \%$} & $3 \%$ & \multirow{3}{*}{10.48} & 421.43 & - & 0.1144 \\
\hline & $4 \%$ & & 493.09 & - & 0.0902 \\
\hline & $5 \%$ & & 609.25 & - & 0.0687 \\
\hline \multirow{3}{*}{$30 \%$} & $3 \%$ & \multirow{3}{*}{9.27} & 714.57 & - & 0.0604 \\
\hline & $4 \%$ & & 837.90 & - & 0.0510 \\
\hline & $5 \%$ & & 1037.69 & - & 0.0413 \\
\hline
\end{tabular}

Table 1: Results for simply supported $(0 / 45 /-45 / 90)_{\mathrm{s}}$ panel (ambient temperature $70^{\circ} \mathrm{F}$ ).

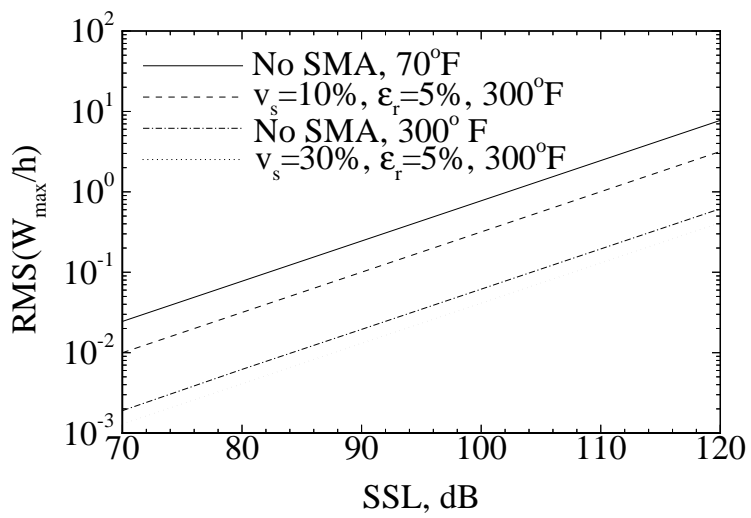

Figure 2: RMS maximum deflection versus sound spectrum level. 


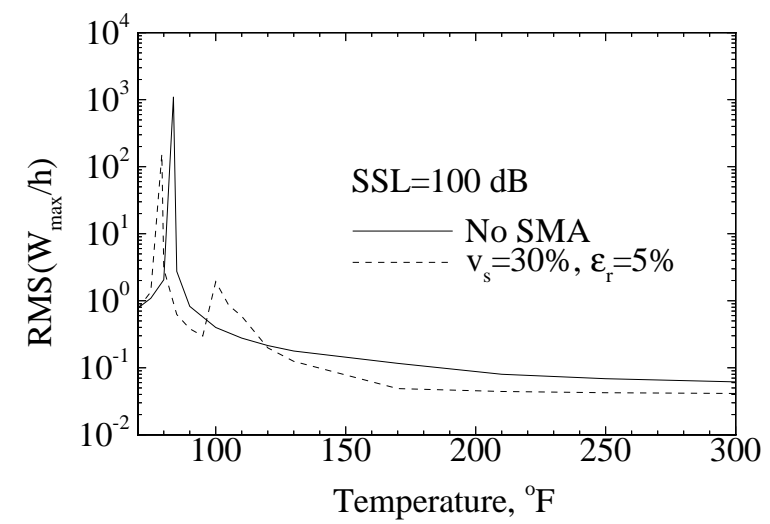

Figure 3: RMS maximum deflection versus temperature.

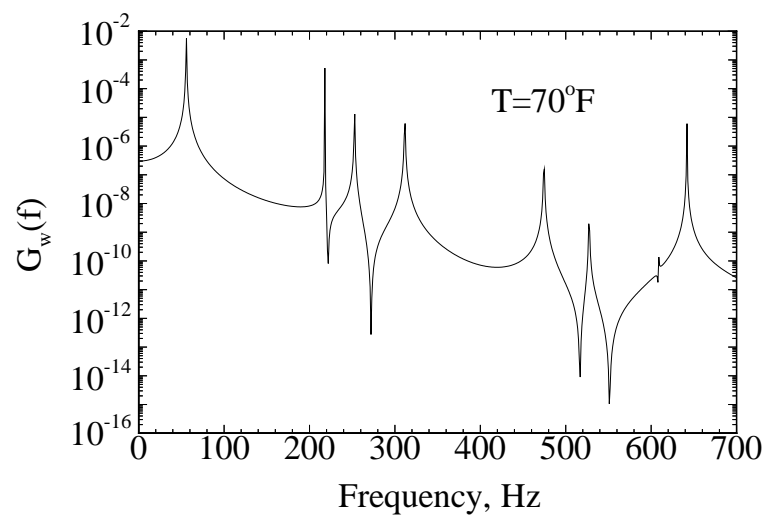

Figure 4: PSD of $W_{\max }$ for panel without SMA at $70^{\circ} \mathrm{F}$.

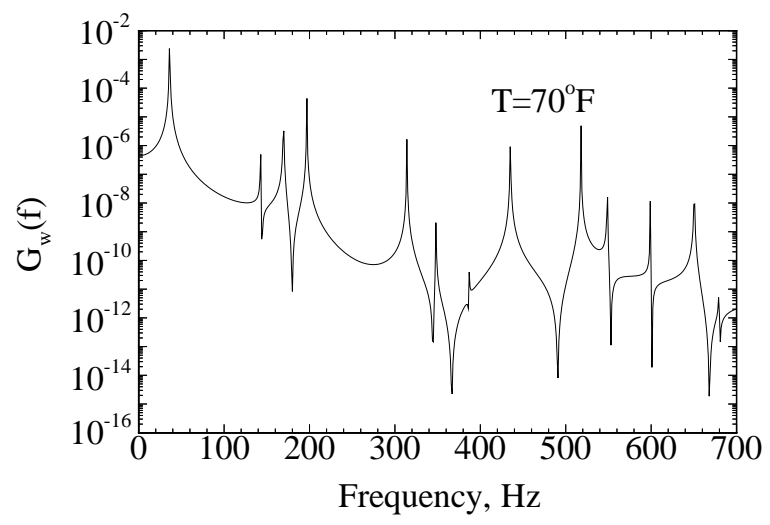

Figure 5: PSD of $W_{\max }$ for $30 \%$ SMA, $5 \%$ initial strain at $70^{\circ} \mathrm{F}$.

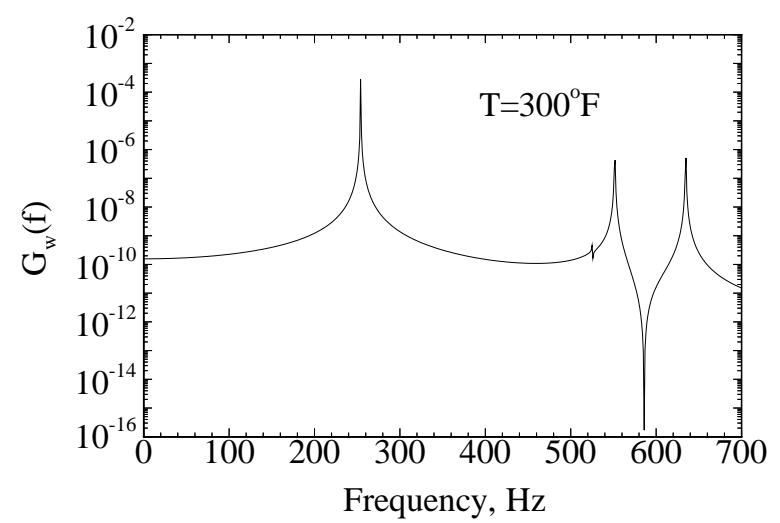

Figure 6: PSD of $W_{\max }$ for $30 \%$ SMA, $5 \%$ initial strain at $300^{\circ} \mathrm{F}$.

\section{Appendix}

\section{Hybrid Lamina Constitutive Relations.}

A representative volume element of a SMA fiberreinforced hybrid composite lamina is shown in Figure 7. The element is taken to be in the plane of the plate. The composite matrix, for example graphiteepoxy, has the principal material directions 1 and 2 and the SMA fiber is embedded in the 1-direction.

In order to derive the constitutive relation for the 1 -direction, it is assumed that a stress $\sigma_{1}$ acts alone on the element $\left(\sigma_{2}=0\right)$ and that the SMA fiber and composite matrix are strained by the same amount, $\epsilon_{1}$ (i.e., plane sections remain plane). The 1 -direction stress-strain relation of the SMA fiber can be described as

$$
\sigma_{1 s}=E_{s}^{*} \epsilon_{1}+\sigma_{r}^{*}, \mathrm{~T}>\mathrm{T}_{\mathrm{s}}
$$

or

$$
\sigma_{1 s}=E_{s}^{*}\left(\epsilon_{1}-\alpha_{s} \triangle T\right), \quad \mathrm{T}<\mathrm{T}_{\mathrm{s}}
$$

where $T_{s}$ is the austenite start temperature and $\alpha_{s}$ is the thermal expansion coeffecient. The Young's modulus $E_{s}^{*}$ and the recovery stress $\sigma_{\mathrm{r}}^{*}$ are temperature dependent, indicated by the superscript $\left(^{*}\right)$. The recovery stress $\sigma_{r}^{*}$ is also dependent on the initial strain $\epsilon_{r}$. For Nitinol, $\sigma_{r}^{*}$ and $\mathrm{E}_{\mathrm{s}}^{*}$ can be determined from Figures 8 and 9 , respectively ${ }^{4}$. Similarly, the one-dimensional stress-strain relation in the 1 -direction for the composite matrix can be expressed as

$$
\sigma_{1 m}=E_{1 m}\left(\epsilon_{1}-\alpha_{1 m} \triangle T\right)
$$

The resultant force in the 1 -direction $\left(\sigma_{2}=0\right)$ is distributed over the SMA fiber and composite matrix and can be written as

$$
\sigma_{1} A_{1}=\sigma_{1 s} A_{s}+\sigma_{1 m} A_{m}
$$


where $\left(\sigma_{1}, A_{1}\right),\left(\sigma_{1 s}, A_{s}\right)$, and $\left(\sigma_{1 m}, A_{1 m}\right)$ are the (stress, cross sectional area) of the entire element, SMA fiber, and composite matrix, respectively. Thus, the average stress $\sigma_{1}$ is

$$
\sigma_{1}=\sigma_{1 s} v_{s}+\sigma_{1 m} v_{m}
$$

where $v_{s}=A_{s} / A_{1}$ and $v_{m}=A_{m} / A_{1}$ are the volume fractions of SMA and composite matrix, respectively. When $T>T_{s}$, the SMA effect is activated and the onedimensional stress-strain relation in the 1-direction becomes

$$
\begin{aligned}
\sigma_{1} & =\left(E_{s}^{*} \epsilon_{1}+\sigma_{r}^{*}\right) v_{s}+E_{1 m}\left(\epsilon_{1}-\alpha_{1 m} \triangle T\right) v_{m} \\
& =E_{1}^{*} \epsilon_{1}+\sigma_{r}^{*} v_{s}-E_{1 m} \alpha_{1 m} v_{m} \triangle T
\end{aligned}
$$

where

$$
E_{1}^{*}=E_{1 m} v_{m}+E_{s}^{*} v_{s}
$$

When $T<A_{s}$, the SMA effect is not activated and the stress $\sigma_{1}$ is

$$
\begin{aligned}
\sigma_{1} & =E_{1}^{*} \epsilon_{1}-\left(E_{s}^{*} \alpha_{s} v_{s}+E_{1 m} \alpha_{1 m} v_{m}\right) \triangle T \\
& =E_{1}^{*}\left(\epsilon_{1}-\alpha_{1} \triangle T\right)
\end{aligned}
$$

where

$$
\alpha_{1}=\frac{\left(E_{1 m} \alpha_{1 m} v_{m}+E_{s}^{*} \alpha_{s} v_{s}\right)}{\left(E_{1 m} v_{m}+E_{s}^{*} v_{s}\right)}
$$

A similar constitutive relation may be derived for the 2-direction by assuming that the applied stress $\sigma_{2}$ acts upon both the fiber and the matrix $\left(\sigma_{1}=0\right)$. Thus, the one-dimensional stress-strain relations in the 2-direction for the SMA fiber and the composite matrix become

$$
\sigma_{2 s}=\sigma_{2}=E_{s}^{*}\left(\epsilon_{2 s}-\alpha_{s} \triangle T\right)
$$

and

$$
\sigma_{2 m}=\sigma_{2}=E_{2 m}\left(\epsilon_{2 m}-\alpha_{2 m} \triangle T\right)
$$

respectively. The recovery stress does not appear in Eq. (A10), since the SMA fiber initial strain $\epsilon_{r}$ and recovery stress $\sigma_{r}^{*}$ are considered to be a 1 -direction effect only.

The total elongation is due to strain in the composite matrix and the SMA fiber and may be written in the form

$$
A_{1} \epsilon_{2}=A_{m} \epsilon_{2 m}+A_{s} \epsilon_{2 s}
$$

Thus, the total strain becomes

$$
\epsilon_{2}=\epsilon_{2 m} v_{m}+\epsilon_{2 s} v_{s}
$$

Since $\sigma_{2}=E_{2}^{*}\left(\epsilon_{2}-\alpha_{2} \Delta T\right)$, Eqs. (A10) and (A11) may be substituted into Eq. (A13) to give

$$
\begin{aligned}
\epsilon_{2} & =\frac{\sigma_{2}}{E_{2}^{*}}+\alpha_{2} \Delta T \\
& =\frac{\sigma_{2} v_{s}}{E_{s}^{*}}+\frac{\sigma_{2} v_{m}}{E_{2 m}}+\left(\alpha_{s} v_{s}+\alpha_{2 m} v_{m}\right) \Delta T
\end{aligned}
$$

Therefore, the modulus and thermal expansion coefficient in the 2-direction become

$$
E_{2}^{*}=\frac{E_{2 m} E_{s}^{*}}{\left(E_{2 m} v_{s}+E_{s}^{*} v_{m}\right)}
$$

and

$$
\alpha_{2}=\alpha_{2 m} v_{m}+\alpha_{s} v_{s}
$$

Expressions for the hybrid composite Poisson's ratios and shear moduli follow from similar derivations.

The constitutive relations for a thin composite lamina with embedded SMA fibers can be derived using a similar engineering approach to give

$$
\begin{aligned}
\left\{\begin{array}{c}
\sigma_{1} \\
\sigma_{2} \\
\tau_{12}
\end{array}\right\} & =\left[\begin{array}{ccc}
Q_{11}^{*} & Q_{12}^{*} & 0 \\
Q_{12}^{*} & Q_{22}^{*} & 0 \\
0 & 0 & Q_{66}^{*}
\end{array}\right]\left\{\begin{array}{c}
\epsilon_{1} \\
\epsilon_{2} \\
\gamma_{12}
\end{array}\right\} \\
& +\left\{\begin{array}{c}
\sigma_{r}^{*} \\
0 \\
0
\end{array}\right\} v_{s}-[Q]_{m}\left\{\begin{array}{c}
\alpha_{1} \\
\alpha_{2} \\
0
\end{array}\right\}_{m} v_{m} \triangle T \\
& =\left[Q^{*}\right]\left\{\begin{array}{c}
\epsilon_{1} \\
\epsilon_{2} \\
\gamma_{12}
\end{array}\right\}+\left\{\begin{array}{c}
\sigma_{r}^{*} \\
0 \\
0
\end{array}\right\} v_{s} \\
& -\left([Q]\left\{\begin{array}{c}
\alpha_{1} \\
\alpha_{2} \\
0
\end{array}\right\}\right)_{m} \triangle T, \quad T>T_{s}
\end{aligned}
$$

and

$$
\left\{\begin{array}{c}
\sigma_{1} \\
\sigma_{2} \\
\tau_{12}
\end{array}\right\}=\left[Q^{*}\right]\left(\left\{\begin{array}{c}
\epsilon_{1} \\
\epsilon_{2} \\
\gamma_{12}
\end{array}\right\}-\left\{\begin{array}{c}
\alpha_{1} \\
\alpha_{2} \\
0
\end{array}\right\} \triangle T\right), \quad T<T_{s}
$$

where $[Q]_{m}$ and $\left[Q^{*}\right]$ are the reduced stiffness matrices of the composite matrix and the composite lamina, respectively. The $\left[Q^{*}\right]$ matrix is temperature dependent and is evaluated using the previously derived relations as

$$
\left(E_{1}^{*}, \nu_{12}\right)=\left(E_{1 m}, \nu_{12 m}\right) v_{m}+\left(E_{s}^{*}, \nu_{s}\right) v_{s}
$$

and

$$
\left(E_{2}^{*}, G_{12}^{*}\right)=\frac{\left(E_{2 m} E_{s}^{*}, G_{12 m} G_{s}^{*}\right)}{\left[\left(E_{2 m}, G_{12 m}\right) v_{s}+\left(E_{s}^{*}, G_{s}^{*}\right) v_{m}\right]}
$$

where the $\nu$ 's are Poisson's ratios and the $G$ 's are the shear moduli. The thermal expansion coefficients $\alpha_{1}$ and $\alpha_{2}$ are derived in Eqs. (A9) and (A16). 


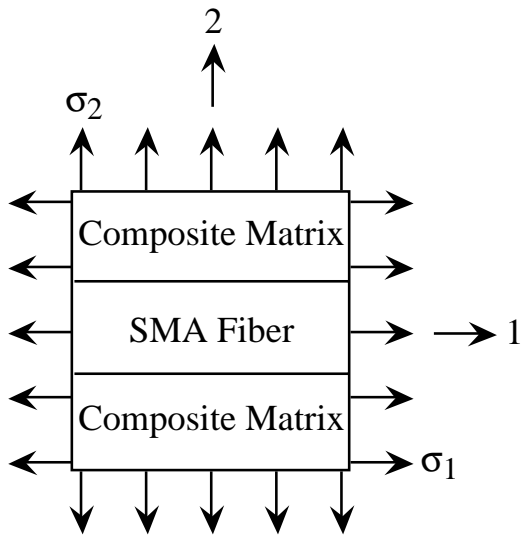

Figure 7: Representative volume element for SMA fiber-reinforced hybrid composite lamina.

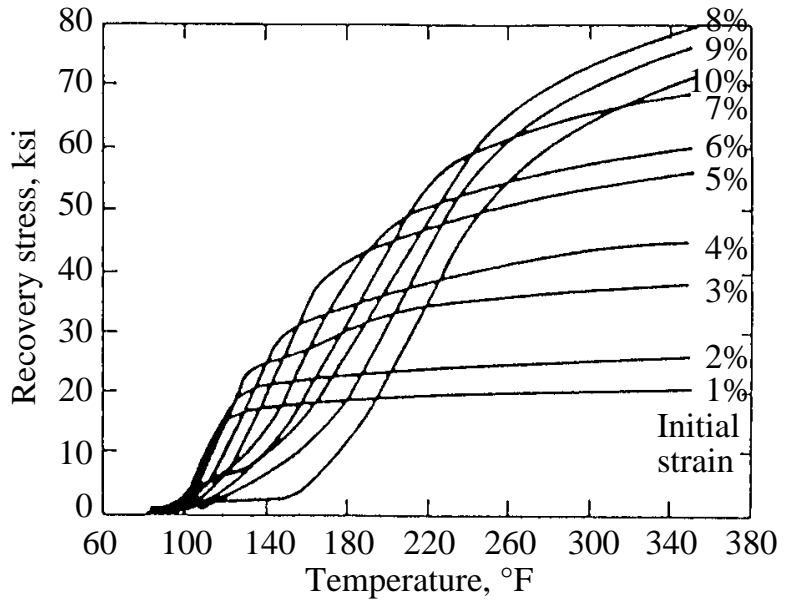

Figure 8: SMA recovery stress versus temperature and initial strain, ref. 4 .

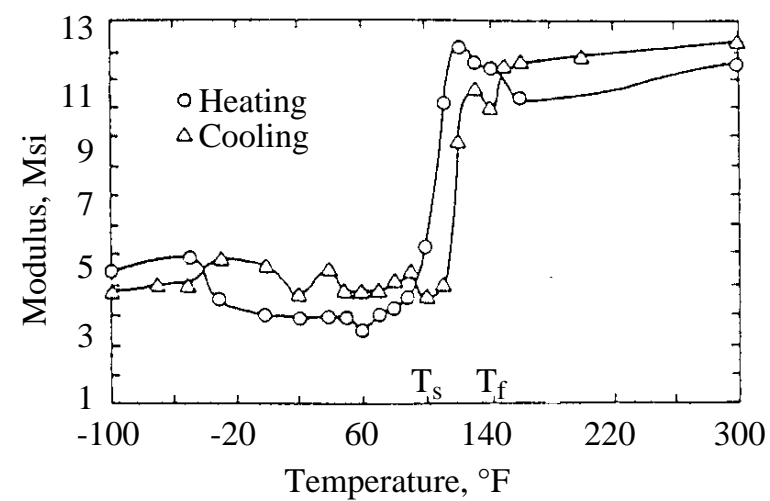

Figure 9: SMA modulus versus temperature, ref. 4. 